\title{
Towards a Business Network Management
}

\author{
Daniel Ritter \\ Technology Development - Process and Network Integration, SAP AG, \\ Dietmar-Hopp-Allee 16, 69190 Walldorf \\ daniel.ritter@sap.com
}

\begin{abstract}
Enterprises are part of value chains consisting of crossenterprise business processes forming large business networks of customers, vendors, partners and competitors. These business process networks run on (technical) integration networks, which are semantically interlinked with the processes, while both are correlated to organizational (social) networks, consisting of technical and business domain experts. The insight into these networks promise a competitive advantage through the visibility into the linked enterprise data. However, this information is hidden in complex, dynamic and heterogeneous enterprise domains.

In this position paper, we introduce the new domain of Business Network Management (BNM), which strives to make business networks visible within network views and sets them into context with each other. For that, we extend the Network Mining (NM) domain by a virtualized network approach towards a BNM. That means automatic discovery, mining and inference capabilities are combined with expert knowledge from different domains to compute the networks and a semantic correlation between entities of different perspectives. We comprehensively define the domain and discuss a system implementation, which we evaluated in a real-world customer case.
\end{abstract}

Keywords: Business Networks, Business Network Management, Business Network Virtualization, Business Processes, Integration Middleware, Linked (Business) Data, Network Mining.

\section{Introduction}

Enterprises are part of value chains consisting of business processes with intra and inter enterprise stakeholders. To remain competitive, enterprises need visibility into their business networks and ideally into relevant parts of partner and customer networks and processes. However, currently the visibility often ends at the borders of systems or enterprises. Business Network Management (BNM) helps to overcome this situation and allows companies to get insight into their technical, social and business relations. However, the relevant information is hidden in complex, dynamic and heterogeneous enterprise domains.

In previous work, we introduced the domain of Network Mining (NM) [10], which allows to discover this information from the different business process and integration domains. The extracted raw data contains the information necessary

G. Poels (Ed.): CONFENIS 2012, LNBIP 139, pp. 149-156, 2013.

(C) IFIP International Federation for Information Processing 2013 
to reconstruct the different network perspectives as well as to semantically link them into one business network [11. An example for a business network is given in Fig. 1, which shows participants in a business process network of a (cross-) enterprise partner network (front) and the corresponding technical network on which these processes are implemented (back). The participants of the business process network represent business artifacts within an enterprise, that are related to participants within a partner network. The participants and relationships are considered complex and contain the underlying business processes, which specify e.g. a business document or goods exchange between related participants. These business processes are actually implemented within the applications and integration capabilities of the enterprises denoting a more technical network, called integration network. This network consists of applications and integration middleware for internal business processes related to processes interacting with business partners like suppliers, transport carriers, dealers.

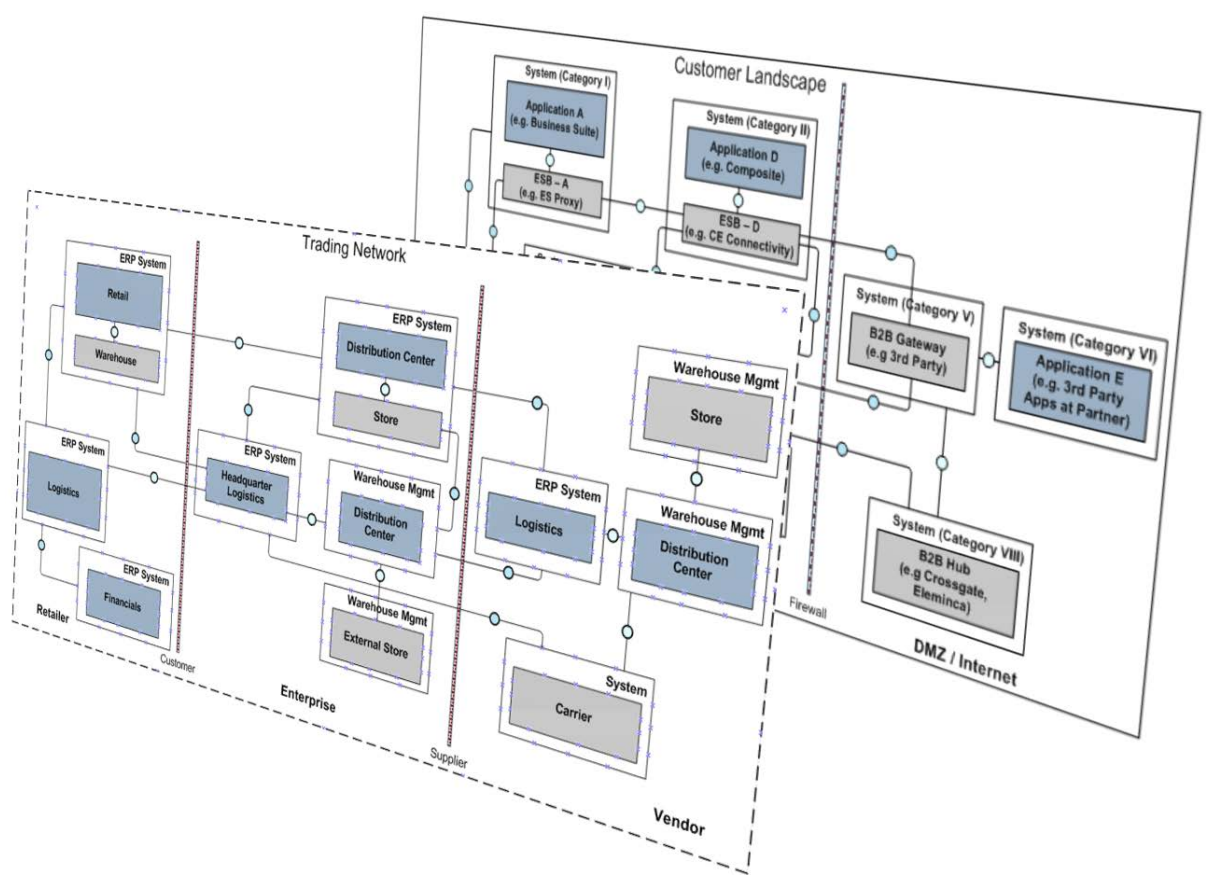

Fig. 1. Sample (cross-) enterprise Business Network showing business participants, denoted as nodes, and business document exchange as edges (front) as well as an integration network perspective (back). Enterprises are characterized by their roles they play within a process.

In this position paper, we introduce a new enterprise and linked data domain, the Business Network Management, which helps enterprises to get insight into their business networks. Based on previous work in the area of Network 
Mining [10, we show how to bridge the gap from the NM raw data towards reconstructed business networks. For that, we use the concept of a virtualized business network, which extends the capabilities of NM from discovery to enrichment and re-deployment of information as required for the management of business networks. While developing the domain towards BNM, we discuss relevant areas for research and set them into context with the state-of-the art up to our knowledge. We conclude with a description of customer cases, which state the current progress of our BNM system.

In section 2 we introduce network virtualization to extend the Network Mining approach. On this foundation we define BNM in section 3 and discuss our prototypical application to real-world enterprise landscapes. We conclude with related work and draw conclusions.

\section{The Virtualized Business Network}

Business Network Virtualization (BNV) is the process of combining network resources and network functionality into a single, software-based administrative entity called (virtual) business network. Within business networks, this allows for efficient utilization, manageability and regulatory compliance. For business networks, we distinguish several levels of virtualization and their options shown in Fig. 2 ,

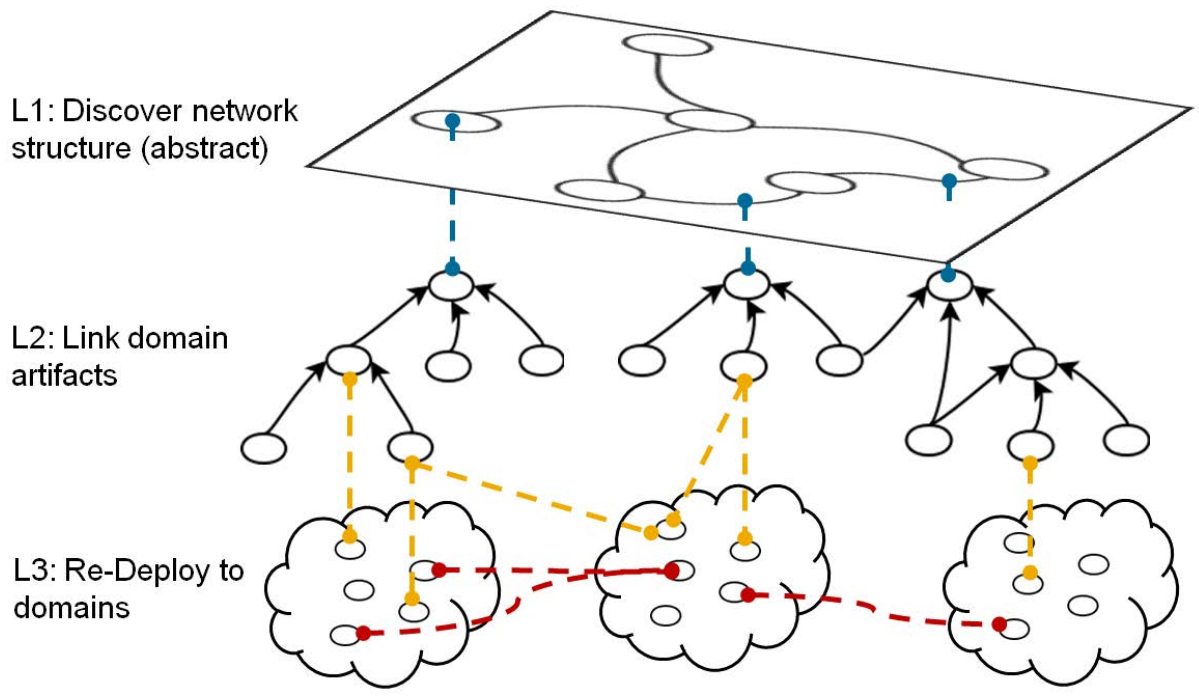

Fig. 2. Sample (cross-) enterprise Business Network showing business participants, denoted as nodes, and business document exchange as edges. Enterprises are characterized by their roles they play within a process. 
The first level (L1) specifies the retrieval and interpretation of business process and integration content as well as operation information, which can be extended as second option by runtime artifacts. Therefore, e.g. integration artifacts are implemented in domain or vendor specific tools, but displayed within the business network. The integration or business errors are fixed within the domainspecific tools. To bring this to the second level (L2), application, design time and operation integration content is encapsulated and generated from the business network. For instance, when the business network is visualized, business process and integration artifacts can be defined on the network as expert knowledge and deployed back to the specific integration technology. Based on level 2, the integration content are enriched by process and information flow models (L3). These models allow a drill-down e.g. to the channel, interface, binding or processing level of the integration technology. As second option to L3, the process and integration flow models are interpreted and executed in one system integrated in the heterogeneous applications and integration technology runtimes.

The levels of virtualization conceptually ground an extended definition of mining Business Networks. The virtualization level L1 corresponds to the first two types of Network Mining (NM), i.e. discovery and conformance, in [10] and covers the discovery, extraction and domain specific analysis of relevant data from dynamic, distributed and heterogeneous enterprise landscapes. Fig. 3(a) shows all three types including network enhancement.

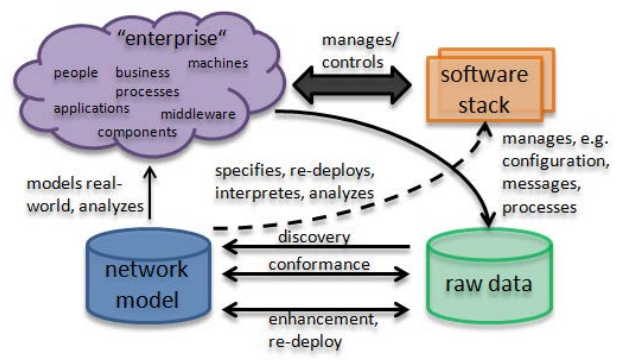

(a) Network Mining as discipline [10]

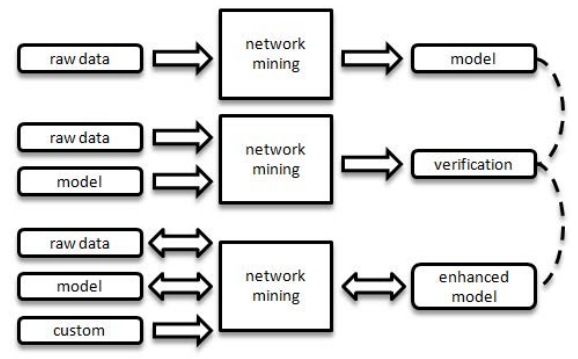

(b) Network Mining Types as Input/Output

Fig. 3. Business Network Mining extended

The third type of network mining is enhancement, which covers re-deployment. Here, the idea is to extend or improve an existing network model using domain expert knowledge about the actual business network. Whereas conformance measures the alignment between model and reality, this type aims at changing or extending the "as-is" model. For instance, the "as-is" model of the network can be refined and extended by introducing new relationships on the network, which results in an integration channel re-deployment within the specific middleware. 
This corresponds to levels L2 and L3 of BNV, which is the most difficult part of $\mathrm{NM}$ and requires a bijective mapping between domain-specific and the (virtual) business network model. In case of external linked data, re-deployment would mean to e.g. post messages into dedicated social media or add new connections to a special profile from an abstract social network view.

Fig. 3(b) summarizes the three NM types in terms of input and output. Techniques for discovery take domain-specific raw data and produce a network model. The discovered models are typically completely diverse like configuration, system landscape, business process models. Conformance checking techniques need that raw data and the network model as input. The output consists of verification information showing differences and commonalities between model and data. Techniques for model enhancement and re-deployment, e.g. repair, extension, need the raw data, the model and optionally expert knowledge as input. The output is an improved or extended model. The custom information can come from various personas which are experts in one of the many domains the raw data comes from. Their knowledge and experience is important and is brought into NM through the custom extensions.

\section{Business Network Management}

The idea of Business Network Management (BNM) is to discover, make visible, monitor (operations) and improve real-world business networks and underlying processes by extracting information from various sources readily available in today's (information) systems. The automated data discovery, conformance checking and enhancement is done by NM resulting in a (virtual) network model. This data is input to inference mechanisms which derive the real "as-is" business network and later a business network spanning across enterprises. With that, BNM aims to ease the end-to-end lifecycle behind integration developments and allows collaboration on different information for faster execution. The network gives a generalized view of an enterprise integration and business landscape.

Today this challenge is addressed with documentation and systems management, but leaves manual work for IT administrators and integration experts to gather a consistent view of the network. The basic steps within BNM are depicted in Fig. 4. The real "as-is" network is computed based on the data from $\mathrm{NM}$ and visualized showing views on business and integration networks as well as their semantic relationships, e.g. processes implemented by middleware systems or applications. Domain experts work on these views by contextualizing, enriching and adapting the network to their needs, e.g. label or group entities. Thereby the experts analyze the "as-is" network and enhance it by e.g. adding new entities. The enhanced network is closer to the "to-be" network, which influences the general "to-be" business process picture derived from BPM or Process Mining (PM) 21]. For that, feedback from the operation on the network is taken into account, e.g. monitoring. 


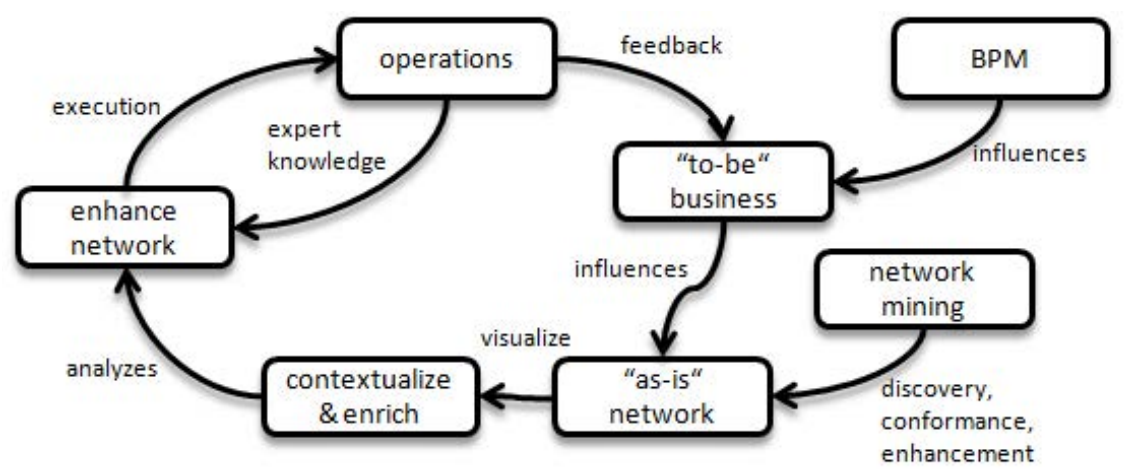

Fig. 4. Business Network Management cycle showing influencing and contributing domains to the "as-is" network computation towards a "to-be" network enhancement

\section{The Business Network Management Prototype}

To demonstrate the "as-is" network computation and assess the approach with customers, we developed a virtualization level L1 prototype, that auto-discovers and graphically represents integration networks. We applied the system to realworld customer landscapes containing mediated communication through middleware systems, e.g. SAP Process Integration (SAP PI) [12, direct connectivity, e.g. web services, and system landscape information, e.g. 13]. An excerpt of the computed integration network from a big manufacturing company is shown in Fig. 5(a) The network consists of participants representing the logical senders and receivers of messages, e.g. application systems and technical endpoints representing a business partner application/server. On a separate screen detail information is shown, e.g. system descriptions or installed products for an application system (not shown). Moreover, the network shows all message exchanges between the selected participants as drill-in on top-level connections, see Fig. 5(b) For each message exchange additional attributes that constitute and describe the message exchange is shown, e.g. technical information like interfaces and transport protocol used or runtime information like last used time.

This application to real-world enterprise landscapes allowed the evaluation of cross-middleware inference, combination of embedded and mediated communication and fragmented information registered in different domains. With that we showed that the BNM approach is valid and resulted in highly reliable and usable results. Moreover, our system is helpful in the everyday work of integration experts, since it gives an overview of the complete "as-is" network, which is very difficult to identify using existing middleware tools. The system reduces the effort to document integration scenarios and helps to answer questions that are difficult to find today. For instance, when combing configuration and runtime data, it is possible to find unused and possibly obsolete interfaces and flows. Hence, with this system, several customers plan upgrade projects of their middleware content, which will substantially save migration time and effort. 


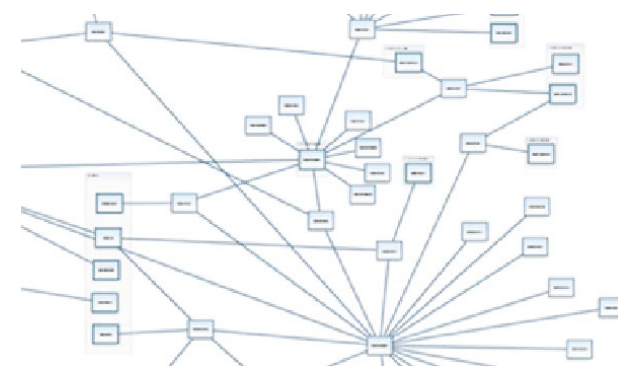

(a) High-level view on an integration network

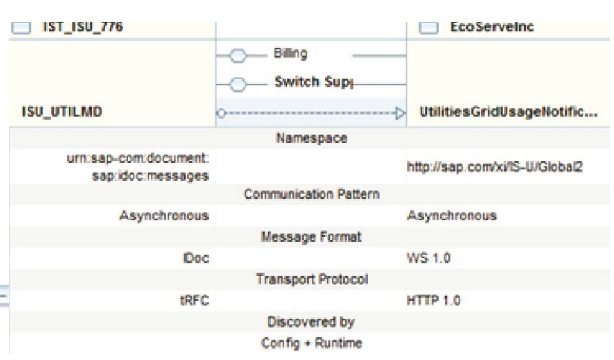

(b) Conversation details

Fig. 5. Integration network visualization showing a view on the network (left) and details of the conversations (right)

\section{Related Work}

This work builds on previous work conducted in [10], which discusses how to come from Network Mining to large scale business networks. The contribution of this paper starts with the definition of Business Network Virtualization, which is used to extend the definition of Business Network towards the Business Network Management.

The most influencing related work is conducted in the area of Process Mining (PM) initiated by 122, which is a relatively young research discipline that sits between computational intelligence and data mining. It has similar requirements for data discovery, conformance and enhancement. However, its approach and goals are different. PM strives to derive Business Process Management (BPM) models from process logs. From that, models are automatically generated and checked. PM as well as BNM complement BPM by making it visible through automated discovery and in case of BNM to set the business processes in a broader context to each other.

Similar to Process Mining, Semantic Business Process Management (SBPM) 8] strives to mine business processes semantics mechanically. For that, an ontological approach [76] is combined with Semantic Web Services (SWS) and BPM. As in the Process Mining case, SBPM and Business Network Management are complementing approaches with combinable technology stacks.

Gaining insight into the network of physical and logical nodes within companies could be a future extension of BNM, but is not primarily relevant for visualizing and operating business networks. This domain is mainly addressed by the IT service management [9] and virtualization community [5].

The linked (web) data research, conducted by Bizer, and Berners-Lee et al. [34], shares similar approaches and methodologies, which have so far neglected linked data within enterprises. 


\section{Discussion and Future Work}

In this position paper, we introduce a new enterprise and linked (business) data domain. We showed how the concept of Business Network Virtualization is used to extend Network Mining towards the Business Network Management. We showed excerpts of a real-world integration enterprise network in our Business Network Center to illustrate how BNM systems could look like.

Future work will be conducted especially for the virtualization levels L2 and L3, i.e. re-deployment and operations on the network, as well as discovering, inferring and making visible further aspects of business networks (for L1). Thus letting them grow to cross-enterprise partner networks in one dimension and towards BPM and network virtualization in the other dimension.

\section{References}

1. van der Aalst, W., et al.: Process Mining Manifesto. Technical Report, IEEE Task Force (2011)

2. van der Aalst, W.: Process Mining: Discovery, Conformance and Enhancement of Business Processes (2011)

3. Bizer, C., Heath, T., Berners-Lee, T.: Linked Data - The Story so Far. International Journal on Semantic Web and Information Systems 5(3), 1-22 (2009)

4. Bizer, C.: The Emerging Web of Linked Data. IEEE Intelligent Systems 24(5), 87-92 (2009)

5. Chowdhury, N.M.M.K., Boutaba, R.: Network virtualization: state of the art and research challenges. IEEE Communications Magazine (2009)

6. Filipowska, A., Hepp, M., Kaczmarek, M., Markovic, I.: Organisational Ontology Framework for Semantic Business Process Management. In: Abramowicz, W. (ed.) BIS 2009. LNBIP, vol. 21, pp. 1-12. Springer, Heidelberg (2009)

7. Hepp, M., Roman, D.: An Ontology Framework for Semantic Business Process Management. Wirtschaftsinformatik, 423-440 (2007)

8. Hepp, M., Leymann, F., Dominigue, J., Wahler, A., Fensel, D.: Semantic Business Process Management - A Vision Towards Using Semantic Web Services for Business Process Management. In: IEEE International Conference on e-Business Engineering (ICEBE), Beijing (2005)

9. O'Neill, P., et al.: Topic Overview - IT Service Management. Technical Report, Forrester Research (2006)

10. Ritter, D.: From Network Mining to Large Scale Business Networks. In: International Workshop on Large Scale Network Analysis (LSNA), WWW Companion, Lyon (2012)

11. Ritter, D., Westmann, T.: Business Network Reconstruction Using Datalog. In: Barceló, P., Pichler, R. (eds.) Datalog 2.0 2012. LNCS, vol. 7494, pp. 148-152. Springer, Heidelberg (2012)

12. SAP Process Integration (2012), http://www.sap.com/germany/plattform/netweaver/components/pi/index.epx

13. Hengevoss, W., Linke, A.: SAP NetWeaver System Landscape Directory - Grundlagen und Praxis. SAP Press (2009) 